\title{
Estudio Teórico de la Hidrólisis de Ésteres Alifáticos y Aromáticos
}

\author{
Silvana C. Caglieri y Héctor R. Macaño \\ Universidad Tecnológica Nacional, Facultad Regional Córdoba, Departamento de Ingeniería \\ Química, Av. Cruz Roja Argentina esquina Maestro López, Ciudad Universitaria (5016), \\ Córdoba - Argentina (e-mail : scaglieri@quimica.frc.utn.edu.ar ; hmacano@quimica.frc.utn.edu.ar)
}

Recibido Oct. 08, 2009; Aceptado Dic. 02, 2009; Versión Final recibida Dic. 10, 2009

\begin{abstract}
Resumen
Se realizó un estudio teórico comparativo de reactividad del acetato de etilo, éster alifático y del benzoato de etilo, éster aromático, frente a la reacción de hidrólisis catalizada por ácido, a través del análisis de los intermediarios de ambas reacciones. Se modelaron y determinaron las geometrías de todas las especies que intervienen en ambas hidrólisis y se calcularon los parámetros geométricos y las energías de los reactivos y productos, empleando dos métodos de estructura electrónica, DFT y AM1. Los valores obtenidos se compararon con datos experimentales y de la literatura observándose una gran concordancia. Siguiendo el mismo procedimiento se determinaron los parámetros geométricos y energías de los intermediarios de reacción, observándose una mayor estabilidad para el intermediario alifático, que reportó una energía inferior comparada con la del aromático. La diferencia de energía entre ambos fue de $34.11 \mathrm{kcal} / \mathrm{mol}$.
\end{abstract}

Palabras claves: hidrólisis ácida, éster alifático, éster aromático, reactividad

\section{Theoretical Study of Aliphatic and Aromatic Esters Hydrolysis}

\begin{abstract}
A comparative theoretical study of reactivity of ethyl acetate hydrolysis, aliphatic ester, and ethyl benzoate hydrolysis, aromatic ester, catalyzed by acid, from the analysis of intermediates in both reactions was carried out. The geometry of all species involved in both hydrolysis was determined and the energies of all reagents and products were calculated, using two methods of electronic structure, DFT and AM1. The calculated values were compared with experimental and literature data finding good agreement. Following the same procedure, the geometric parameters and energies of reaction intermediates were determined, observing greater stability for the aliphatic intermediate, with lower energy compared with the aromatic. The energy difference between them was $34.11 \mathrm{kcal} / \mathrm{mol}$.
\end{abstract}

Keywords: acid hydrolysis, aliphatic ester, aromatic ester, reactivity 


\section{INTRODUCCIÓN}

El estudio teórico de la hidrólisis ácida de los ésteres alifáticos y aromáticos, cuyos productos son alcoholes y ácidos orgánicos, es de gran interés, no sólo por la utilidad de los productos de reacción dentro de la industria química, sino que desde el punto de vista ambiental dicha reacción constituye una vía para la degradación de los poliésteres, polímeros sintéticos conformados por monómeros ésteres. Han sido reportados estudios sobre síntesis y caracterización de poliésteres alifáticos biodegradables (Pepic et al., 2008), investigaciones sobre biodegradabilidad de copoliésteres alifático-aromático (Chen et al., 2008) y sobre el mecanismo de reacción de la hidrólisis de poliésteres alifáticos (Partini y Pantani, 2007). Todos estos estudios coinciden en que los poliésteres formados por monómeros aromáticos poseen mejores propiedades mecánicas y físicas, pero son menos biodegradables y más resistentes a la degradación por hidrólisis que aquellos poliésteres que sólo contienen monómeros alifáticos, esto lleva a la necesidad de sintetizar copoliésteres con unidades alifáticas y aromáticas para poder satisfacer ambos requerimientos.

Los ésteres se hidrolizan lentamente ya que poseen grupos salientes muy básicos y además el agua es un nucleófilo débil. El aumento de la velocidad de la hidrólisis se puede conseguir con el agregado de un ácido como catalizador. Para que un catalizador aumente la velocidad de una reacción debe aumentar la velocidad del paso lento o paso determinante de la misma. La hidrólisis es una reacción de sustitución nucleofílica sobre carbono insaturado, el ácido que actúa como catalizador, primero protona el oxígeno del carbonilo y posteriormente se produce el ataque nucleofílico del agua para formar el intermediario tetraédrico, etapa determinante de la velocidad de reacción. El ácido aumenta la velocidad de formación del intermediario tetraédrico al protonar el oxígeno del carbonilo, porque un grupo carbonilo protonado es más susceptible de ser atacado por un nucleófilo que un grupo carbonilo no protonado.

Se han llevado a cabo estudios teóricos sobre la hidrólisis de ésteres alifáticos catalizada con ácidos (Hori et al., 2007), con bases (Haeffner et al., 1999) y aplicación de diversos métodos computacionales para estudiar la velocidad de dicha reacción mediante catálisis alcalina (Zhang et al., 2005). Tanto estos estudios, como trabajos experimentales y estudios cinéticos (Calvar et al., 2006) concuerdan en que dicha reacción transcurre a través de la formación de un intermediario tetraédrico. En este artículo se presenta un estudio teórico comparativo de la hidrólisis ácida del acetato de etilo y del benzoato de etilo, cuyos productos de reacción son el etanol y los ácidos acético y benzoico respectivamente, empleando la Química Computacional como herramienta para estudiar las estructuras y propiedades de compuestos orgánicos y predecir comportamientos de reacciones químicas.

\section{METODOLOGìA}

Para poder comparar la reactividad de ambos ésteres frente a la hidrólisis, se diseñaron y optimizaron las estructuras de todas las especies que intervienen en las reacciones, determinándose los parámetros geométricos óptimos correspondientes, calculando además las energías mínimas de todos los compuestos, reactivos y productos, que participan en las reacciones como así también la energía de los respectivos intermediarios. Para realizar estos cálculos se emplearon métodos de estructura electrónica. Estos métodos dentro de la química computacional, utilizan las leyes de la mecánica cuántica como base para los cálculos, mediante la resolución de la ecuación de Schrödinger, que independiente del tiempo toma la forma de la siguiente ecuación:

$H \Psi_{(r)}=E \Psi_{(r)}$

Siendo $\mathrm{H}$ una función diferencial que incluye la energía cinética y potencial de núcleos y electrones, denominada operador hamiltoniano, $\Psi$ la función de onda molecular y $E$ energía. La solución que obtiene el menor valor de energía es la que corresponde al estado basal.

Dentro de los métodos de estructura electrónica, se empleó el método DFT basado en la teoría de funcionales de densidad (Parr y Yang, 1989), el cual incluye efectos de correlación electrónica y 
dentro de este se empleó el B3LYP (Becke, 1997). Este método denominado ab-initio, considera toda la estructura atómica sin introducir parámetros empíricos en la resolución de la ecuación de Schrödinger. Se optó por la función de base $3-21 G^{*}$ que indica que se utilizan tres funciones gaussianas para representar los orbitales atómicos internos, mientras que los externos, capa de valencia, se representan mediante dos funciones para la parte contraída y una para la parte difusa, más una función de polarización para átomos pesados, indicada mediante el *, que representa el efecto de los orbitales tipo d.

También para calcular las energías mínimas de todas las moléculas que intervienen en ambas reacciones se empleó el método semiempírico AM1, Austin Model 1 (Dewart et al., 1985), que utiliza parámetros derivados de resultados experimentales para simplificar los cálculos cuánticos y reducir su coste computacional. Este método omite los cálculos relativos a los electrones de capa interna, que los trata como si formasen parte del núcleo y se centra únicamente en los electrones externos, considerando a éstos electrones los responsables de la reactividad. Todos los cálculos se realizaron empleando el programa Gaussian03 (Frisch et al., 2004).

\section{RESULTADOS Y DISCUSIÓN}

Ambas hidrólisis, tanto la del acetato de etilo, éster alifático como la del benzoato de etilo, éster aromático, transcurren por el mismo mecanismo de reacción. En las Fig.1 y Fig. 2 se describen las etapas a través de las cuales se llevan a cabo las hidrólisis ácidas de ambos ésteres. Ambas reacciones son reversibles. La hidrólisis ácida de los ésteres es la reacción inversa a la esterificación. En la Fig. 3 se observan las estructuras de los intermediarios de reacción, alifático y aromático de ambas hidrólisis.

La Tabla 1 reporta los resultados obtenidos de la aplicación de los métodos de cálculo mencionados, es decir en la misma se pueden observar las longitudes de enlace (r), los ángulos de enlace $(\theta)$, los momentos dipolares $(\mu)$ y la energía $(E)$ de los reactivos y productos que intervienen en cada una de las reacciones motivo de estudio. Se observa una marcada concordancia entre los valores de energía que resultan de los cálculos teóricos indicados con $\mathrm{E}$ en $\mathrm{kcal} / \mathrm{mol}$ y los valores obtenidos de bibliografía indicados con $\Delta \mathrm{H}_{\mathrm{f}}^{\circ}$ en $\mathrm{kcal} / \mathrm{mol}$ en fase gaseosa (Yaws, 1999).

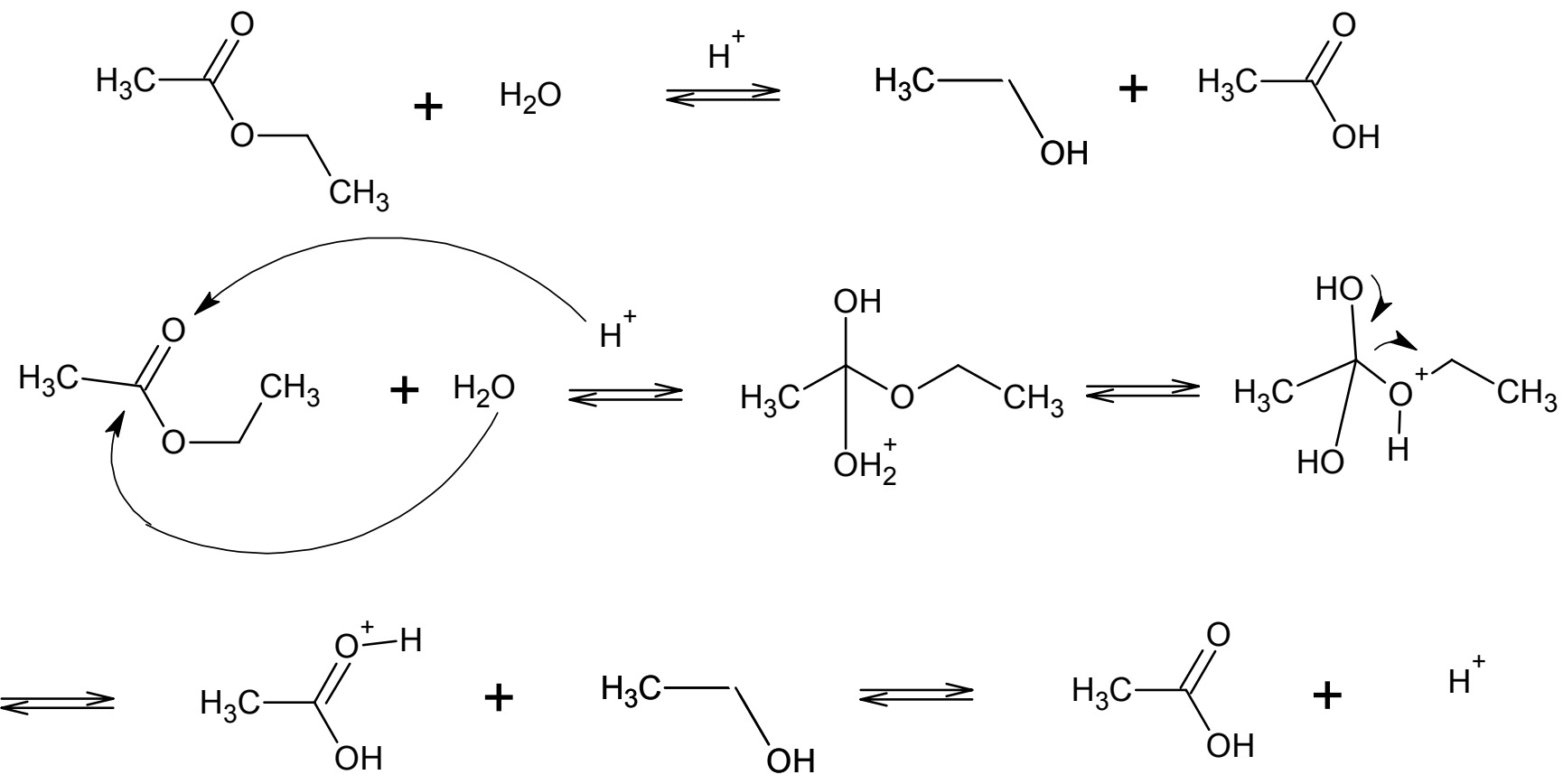

Fig. 1: Mecanismo de la hidrólisis ácida del acetato de etilo 

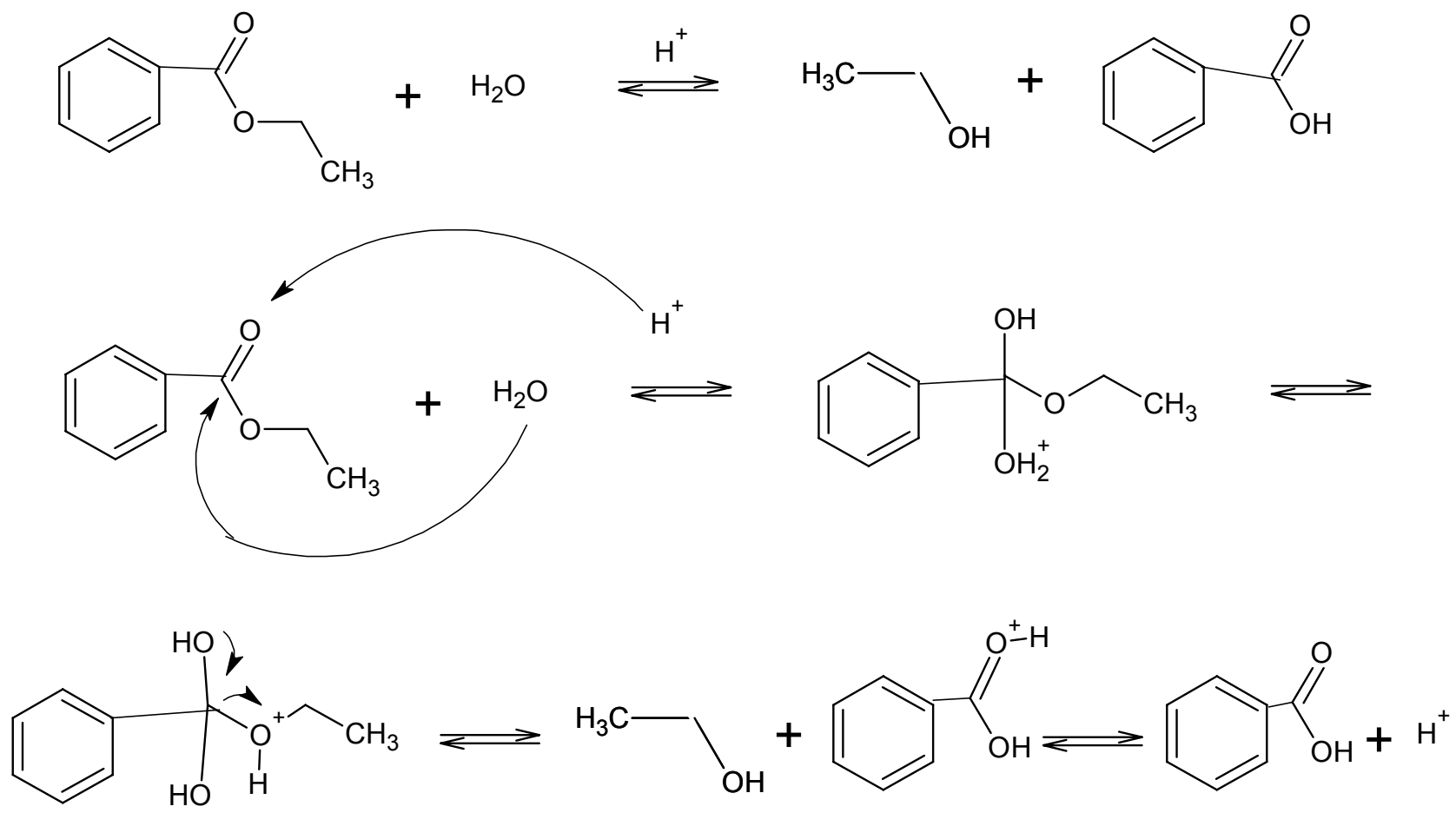

Fig. 2: Mecanismo de la hidrólisis ácida del benzoato de etilo

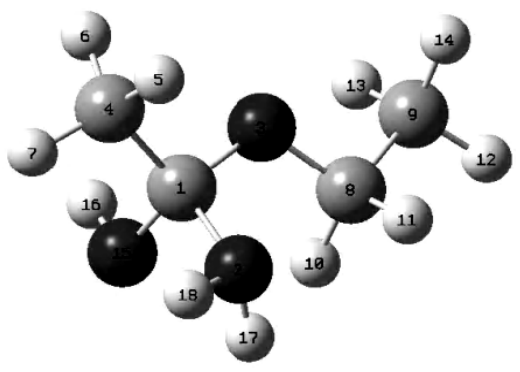

Intermediario Alifático

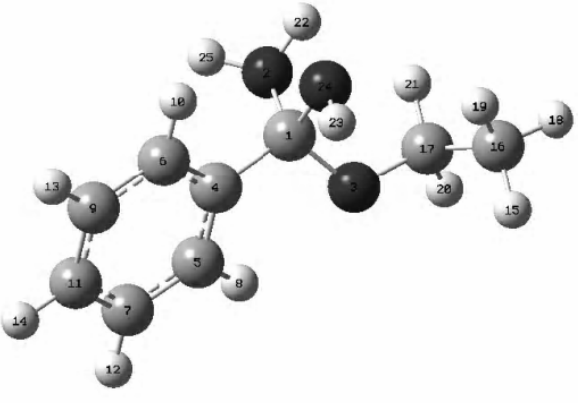

Intermediario Aromático

Fig. 3: Estructura intermediarios alifático y aromático

Tabla 1: Parámetros geométricos y energías de reactivos y productos

\begin{tabular}{|c|c|c|c|c|c|c|}
\hline & Agua & $\begin{array}{c}\text { Ácido } \\
\text { Benzoico }\end{array}$ & $\begin{array}{c}\text { Benzoato } \\
\text { de Etilo }\end{array}$ & Etanol & $\begin{array}{c}\text { Ácido } \\
\text { acético }\end{array}$ & $\begin{array}{c}\text { Acetato } \\
\text { de etilo }\end{array}$ \\
\hline $\mathrm{r}(\mathrm{O}-\mathrm{H})$ & $0.997 \AA$ & $0.99 \AA$ & - & $0.99 \AA$ & $0.99 \AA$ & - \\
\hline$\theta(\mathrm{H}-\mathrm{O}-\mathrm{H})$ & $103.9^{\circ}$ & - & - & - & - & - \\
\hline $\mathrm{r}(\mathrm{C}-\mathrm{O})$ & - & - & $1.43 \AA$ & $1.46 \AA$ & - & $1.48 \AA$ \\
\hline $\mathrm{r}(\mathrm{C}-\mathrm{C})$ & - & - & $1.53 \AA$ & $1.53 \AA$ & - & $1.53 \AA$ \\
\hline $\mathrm{r}(\mathrm{C}-\mathrm{H})$ & - & - & $1.09 \AA$ & $1.097 \AA$ & $1.09 \AA$ & $1.09 \AA$ \\
\hline$\theta(\mathrm{Csp}-\mathrm{O}-\mathrm{H})$ & - & - & - & $107.7^{\circ}$ & - & - \\
\hline
\end{tabular}


Tabla 1: (continuaciòn)

\begin{tabular}{|c|c|c|c|c|c|c|}
\hline$\theta(\mathrm{H}-\mathrm{C}-\mathrm{H})$ & - & - & $109^{\circ}$ & $109.5^{\circ}$ & $109.2^{\circ}$ & $109^{\circ}$ \\
\hline $\mathrm{r}(\mathrm{C}=\mathrm{O})$ & - & $1.23 \AA$ & $1.23 \AA$ & - & $1.22 \AA$ & $1.23 \AA$ \\
\hline $\mathrm{r}\left(\mathrm{Csp}^{3}-\mathrm{C}=\right)$ & - & - & - & - & $1.51 \AA$ & $1.51 \AA$ \\
\hline $\mathrm{r}(=\mathrm{C}-\mathrm{O})$ & - & $1.38 \AA$ & $1.38 \AA$ & - & $1.38 \AA$ & $1.38 \AA$ \\
\hline$\theta(\mathrm{O}-\mathrm{C}=\mathrm{O})$ & - & $122.35^{\circ}$ & $123.1^{\circ}$ & - & $122.46^{\circ}$ & $123.23^{\circ}$ \\
\hline$\theta(=\mathrm{C}-\mathrm{O}-\mathrm{H})$ & - & $108.1^{\circ}$ & - & - & $107.9^{\circ}$ & - \\
\hline$\theta(\mathrm{C}-\mathrm{C}(=)-\mathrm{O})$ & - & - & - & - & $127.4^{\circ}$ & $127.47^{\circ}$ \\
\hline $\mathrm{r}(\mathrm{C}-\mathrm{C})$ Anillo & - & $1.39 \AA$ & $1.39 \AA$ & - & - & - \\
\hline $\mathrm{r}(\mathrm{C}-\mathrm{H})$ Anillo & - & $1.08 \AA$ & $1.08 \AA$ & - & - & - \\
\hline $\mathrm{r}(\mathrm{Canillo-C}=)$ & - & $1.47 \AA$ & $1.48 \AA$ & - & - & - \\
\hline$\theta(\mathrm{Canillo-C}(=)-\mathrm{O})$ & - & $125.8^{\circ}$ & $125.3^{\circ}$ & - & - & - \\
\hline $\mathrm{E} \mathrm{Kcal/mol}$ & -59.25 & $-68,1$ & -67.22 & -56.9 & -102 & -102.3 \\
\hline$\Delta \mathrm{H}_{\mathrm{f}}^{\circ} \mathrm{Kcal} / \mathrm{mol}$ & -57.84 & -69.43 & -67.94 & $-56,17$ & -104.03 & -105.96 \\
\hline
\end{tabular}

En las Tablas 2 y 3 se muestran los parámetros geométricos, valores de longitudes y ángulos de enlaces de los intermediarios alifáticos y aromáticos respectivamente.

Tabla 2: Parámetros geométricos del intermediario alifático

\begin{tabular}{|l|c|c|c|c|c|}
\hline Longitud de enlace & Valor & Angulo de enlace & Valor & Energía & Valor \\
\hline$r\left(\mathrm{O}_{15}-\mathrm{H}_{16}\right)$ & $0.963 \AA$ & $\theta\left(\mathrm{H}_{16}-\mathrm{O}_{15}-\mathrm{C}_{1}\right)$ & $108.356^{\circ}$ & $\mathrm{E}(\mathrm{kcal} / \mathrm{mol})$ & 21.08 \\
\hline $\mathrm{r}\left(\mathrm{C}_{1}-\mathrm{C}_{4}\right)$ & $1.521 \AA$ & $\theta\left(\mathrm{C}_{1}-\mathrm{C}_{4}-\mathrm{H}_{5}\right)$ & $109.211^{\circ}$ & & \\
\hline $\mathrm{r}\left(\mathrm{C}_{1}-\mathrm{O}_{2}\right)$ & $1.480 \AA$ & $\theta\left(\mathrm{C}_{1}-\mathrm{O}_{2}-\mathrm{H}_{17}\right)$ & $108.589^{\circ}$ & & \\
\hline $\mathrm{r}\left(\mathrm{C}_{9}-\mathrm{C}_{8}\right)$ & $1.511 \AA$ & $\theta\left(\mathrm{C}_{8}-\mathrm{O}_{3}-\mathrm{C}_{1}\right)$ & $115.804^{\circ}$ & & \\
\hline$r\left(\mathrm{C}_{4}-\mathrm{H}\right)$ & $1.114 \AA$ & $\theta\left(\mathrm{O}_{3}-\mathrm{C}_{1}-\mathrm{O}_{2}\right)$ & $109.979^{\circ}$ & & \\
\hline$r\left(\mathrm{C}_{9}-\mathrm{H}\right)$ & $1.115 \AA$ & $\theta\left(\mathrm{H}_{11}-\mathrm{C}_{8}-\mathrm{O}_{3}\right)$ & $108.719^{\circ}$ & & \\
\hline$r\left(\mathrm{C}_{8}-\mathrm{H}\right)$ & $1.122 \AA$ & $\theta\left(\mathrm{H}_{13}-\mathrm{C}_{9}-\mathrm{C}_{8}\right)$ & $110.578^{\circ}$ & & \\
\hline$r\left(\mathrm{C}_{1}-\mathrm{O}_{3}\right)$ & $1.410 \AA$ & $\theta\left(\mathrm{H}_{7}-\mathrm{C}_{4}-\mathrm{C}_{1}\right)$ & $108.745^{\circ}$ & & \\
\hline$r\left(\mathrm{C}_{8}-\mathrm{O}_{3}\right)$ & $1.428 \AA$ & & & & \\
\hline$r\left(\mathrm{C}_{1}-\mathrm{O}_{15}\right)$ & $1.407 \AA$ & & & & \\
\hline$r\left(\mathrm{O}_{2}-\mathrm{H}_{17}\right)$ & $0.968 \AA$ & & & & \\
\hline
\end{tabular}

En ambos intermediarios el carbono central tetraédrico está unido a tres oxígenos y a un carbono. La longitud de enlace $r\left(C_{1}-C_{4}\right)$ en el intermediario alifático es de $1.521 \AA$, superior a la longitud $r\left(C_{1}-\right.$ $\mathrm{C}_{4}$ ) de $1.508 \AA$ que presenta el intermediario aromático. El anillo bencénico es un grupo más voluminoso que el metilo, pero es plano y proporciona un carbono hibridado $\mathrm{sp}^{2}$ para enlazarse al carbono central tetraédrico, esto demuestra su mayor proximidad al carbono central tetraédrico comparado con el metilo que si bien es un grupo más pequeño, proporciona un carbono hibridado $\mathrm{sp}^{3}$ para enlazarse con dicho carbono, de allí que se encuentre más alejado. La existencia y proximidad de un grupo más voluminoso ocasiona un mayor impedimento estérico y los cálculos de energía a nivel teórico indican que el intermediario alifático es $34.11 \mathrm{kcal} / \mathrm{mol}$ más estable que el intermediario aromático. 
Tabla 3: Parámetros geométricos del intermediario aromático

\begin{tabular}{|l|c|l|l|l|c|}
\hline \multicolumn{1}{|c|}{ Longitud de enlace } & Valor & Angulo de enlace & \multicolumn{1}{|c|}{ Valor } & \multicolumn{1}{c|}{ Energía } & Valor \\
\hline$r\left(\mathrm{O}_{2}-\mathrm{H}_{22}\right)$ & $1.010 \AA$ & $\theta\left(\mathrm{C}_{1}-\mathrm{O}_{2}-\mathrm{H}_{22}\right)$ & $103.924^{\circ}$ & $\mathrm{E}(\mathrm{kcal} / \mathrm{mol})$ & 55.19 \\
\hline $\mathrm{r}\left(\mathrm{C}_{1}-\mathrm{C}_{4}\right)$ & $1.508 \AA$ & $\theta\left(\mathrm{C}_{16}-\mathrm{C}_{17}-\mathrm{O}_{3}\right)$ & $110.484^{\circ}$ & & \\
\hline $\mathrm{r}\left(\mathrm{C}_{1}-\mathrm{O}_{2}\right)$ & $1.445 \AA$ & $\theta\left(\mathrm{O}_{3}-\mathrm{C}_{1}-\mathrm{O}_{2}\right)$ & $110.341^{\circ}$ & & \\
\hline $\mathrm{r}(\mathrm{C}-\mathrm{C})$ anillo & $1.397 \AA$ & $\theta\left(\mathrm{H}_{19}-\mathrm{C}_{16}-\mathrm{C}_{17}\right)$ & $110.307^{\circ}$ & & \\
\hline $\mathrm{r}(\mathrm{C}-\mathrm{H})$ anillo & $1.099 \AA$ & $\theta\left(\mathrm{O}_{24}-\mathrm{C}_{1}-\mathrm{O}_{3}\right)$ & $107.333^{\circ}$ & & \\
\hline $\mathrm{r}\left(\mathrm{C}_{17}-\mathrm{H}\right)$ & $1.120 \AA$ & $\theta\left(\mathrm{H}_{10}-\mathrm{C}_{6}-\mathrm{C}_{4}\right)$ & $119.719^{\circ}$ & & \\
\hline $\mathrm{r}\left(\mathrm{C}_{16}-\mathrm{H}\right)$ & $1.116 \AA$ & $\theta\left(\mathrm{C}_{6}-\mathrm{C}_{4}-\mathrm{C}_{1}\right)$ & $119.176^{\circ}$ & & \\
\hline $\mathrm{r}\left(\mathrm{C}_{1}-\mathrm{O}_{3}\right)$ & $1.411 \AA$ & & & & \\
\hline $\mathrm{r}\left(\mathrm{C}_{17}-\mathrm{O}_{3}\right)$ & $1.426 \AA$ & & & & \\
\hline $\mathrm{r}\left(\mathrm{C}_{1}-\mathrm{O}_{24}\right)$ & $1.406 \AA$ & & & & \\
\hline $\mathrm{r}\left(\mathrm{O}_{24}-\mathrm{H}_{23}\right)$ & $0.965 \AA$ & & & & \\
\hline$r\left(\mathrm{C}_{17}-\mathrm{C}_{16}\right)$ & $1.512 \AA$ & & & & \\
\hline
\end{tabular}

Si analizamos el mecanismo de la reacción de sustitución nucleofílica catalizada por ácido, luego de la protonación del oxígeno del carbonilo debida al medio, el carbono del carbonilo experimenta una deficiencia electrónica que en el caso del acetato de etilo está compensada por el efecto inductivo $\mathrm{sp}^{3} \rightarrow \mathrm{sp}^{2}$ ejercido por el grupo metilo y en el caso del benzoato de etilo, la densidad de carga positiva puede deslocalizarse en el anillo bencénico. Esta deslocalización de la carga compensa más la deficiencia electrónica que el efecto debido a la diferencia de hibridación, por lo tanto en el caso del acetato de etilo, el carbono del carbonilo está más susceptible a ser atacado por el agente nucleofílico, siendo por lo tanto mayor la reactividad de este éster frente a la hidrólisis catalizada por ácidos. Este análisis se convalida al comparar las respectivas energías de activación $\left(E_{a}\right)$, obteniéndose las mismas a partir de las especies protonadas. Para la hidrólisis ácida del acetato de etilo se obtuvo una $E_{a}=16.39 \mathrm{kcal} / \mathrm{mol}$, valor que se aproxima al de $15.6 \mathrm{kcal} / \mathrm{mol}$ obtenido experimentalmente (Kirby, 1972) y para la hidrólisis ácida del benzoato de etilo se obtuvo una $E_{a}=$ $24.45 \mathrm{kcal} / \mathrm{mol}$, valor que se aproxima al de $23.82 \mathrm{kcal} / \mathrm{mol}$ calculado a partir del valor experimental de $19.26 \mathrm{kcal} / \mathrm{mol}$, reportado para la reacción inversa de esterificación (Pipus et al., 2000).

\section{CONCLUSIONES}

De acuerdo a los resultados y discusión presentados en este trabajo, el intermediario tetraédrico alifático resulta ser más estable que el aromático, por lo tanto el acetato de etilo presenta una mayor reactividad frente a la hidrólisis ácida, comparado con el benzoato de etilo, presentando este último una menor velocidad de reacción. La facilidad con que los ésteres alifáticos se hidrolizan coincide con la aplicación de esta reacción en la degradación de poliésteres alifáticos.

La existencia de una marcada concordancia entre los resultados teóricos obtenidos y los datos bibliográficos, valida el empleo de los métodos teóricos DFT y AM1 como herramientas para el estudio de la estructura de los reactivos y productos que participan del sistema químico planteado. La aproximación entre los resultados teóricos obtenidos y datos experimentales, valida el empleo de dichos métodos para estimar la cinética del sistema químico planteado.

\section{REFERENCIAS}

Becke, A.D.; Density-functional thermochemistry V. Systematic optimization of exchange-correlation functionals, J. Chem. Phys.: 107, 8554-8560 (1997). 
Calvar, N., B. González y A. Domínguez, Esterification of acetic acid with ethanol: Reaction kinetics and operation in a packed bed reactive distillation column, Chemical Engineering and Processing: 46, 1317-1323 (2007).

Chen, Y., L. Tan, L. Chen, Y.Yang y X. Wang, Study on Biodegradable aromatic/aliphatic copolyesters, Brazilian Journal of Chemical Engineering: 25, 321-335 (2008).

Dewar, M.J.S., E.G.Zoebisch, E.F.Healy y J.J.P.Stewart; AM1: A New General Purpose Quantum Mechanical Molecular Model, J. Am. Chem.Soc.:107, 3902-3909 (1985).

Frisch, M.J., G. W. Trucks, y otros 80 autores; Gaussian 03, Revision E.01; Gaussian, Inc., Wallingford CT, Pittsburgh, USA (2004).

Haeffner, F., C.H. Hu, T. Brinck y T. Nori; The catalytic effect of water in basis hydrolysis of methyl acetate a theoretical study, J. Mol. Struct.Theochem: 459, 85-93 (1999).

Hori, K., Y. Ikenaga, K. Arata, T. Takahashi, K. Kasai, Y. Noguchi, M. Sumimoto y H. Yamamoto; Theoretical study on the reaction mechanism for the hydrolysis of esters and amides under acidic conditions, Tetrahedron: 63, 1264-1269 (2007).

Kirby, A.J., Hydrolysis and Formation of Esters of Organic Acids, In Comprehensive Chemical Kinetics, pp 57-161 Elsevier Publishing Company, Amsterdam, The Netherlands (1972).

Parr, R.G. y W. Yang; Density-functional theory of atoms and molecules, Oxford University Press.Nueva York (1989).

Partini M. y R. Pantani, FTIR analysis of hydrolysis in aliphatic polyesters, Polymer Degradation and Stability 92, 1491-1497 (2007).

Pepic, D., E. Zagaz, M.Zigon, A.Krzan, M. Kunaver y J. Djonlagic; Synthesis and characterization of biodegradable aliphatic copolyesters with poly (ethylene oxide) soft segments, European Polymer Journal: 44,904-917 (2008)

Pipus,G., I. Plazl y T. Koloini; Esterification of benzoic acid in microwave tubular flor reactor, Chemical Engineering Journal: 76,239-245 (2000).

Yaws , C.L., Chemical Properties Handbook, Mc Graw Hill, Nueva York (1999).

Zhang, H., X. Qu y H. Ando; A simple method for reaction rate prediction of ester hydrolysis, J. Mol. Struct.Theochem: 725, 31-37 (2005). 